\title{
Preparação e caracterização estrutural de materiais cerâmicos obtidos via processo freeze-casting
}

\author{
(Processing and structural evaluation of ceramic materials \\ prepared by freeze-casting method) \\ D. F. Souza *, E. H. M. Nunes, D. C. L. Vasconcelos, W. L. Vasconcelos \\ Departamento de Engenharia Metalúrgica e de Materiais, Universidade Federal de Minas Gerais, \\ Av. Presidente Antônio Carlos 6627, Belo Horizonte, MG, Brasil 31270-901 \\ *souzadf@outlook.com
}

\begin{abstract}
Resumo
Cerâmicas com porosidade controlada apresentam elevado potencial de aplicação como membranas para separação gasosa e processos de dessalinização. As propriedades e o desempenho desses materiais estão diretamente relacionados ao seu processo de fabricação. Assim, é de vital importância que as variáveis do processo utilizado sejam conhecidas e controladas. Neste trabalho, cerâmicas porosas à base de alumina foram obtidas por meio do processo freeze-casting. Foram utilizados terc-butanol como solvente e ácido cítrico como dispersante. As amostras foram fabricadas a partir de suspensões com concentrações de alumina variando entre 15 e 30\% em volume. Elas apresentaram redução linear da porosidade total (entre 48 e $73 \%$ ) e porosidade fechada inferior a 2\%. A resistência à flexão desses materiais variou entre 3,6 $\pm 0,7$ e 21,9 $\pm 0,6 \mathrm{MPa}$. Ensaios de microscopia eletrônica de varredura e microtomografia de raios X revelaram que as amostras apresentaram elevada conectividade de poros e orientação preferencial de poros. As alterações nas condições de processamento resultaram em materiais com estruturas porosas distintas, demonstrando a versatilidade do processo freeze-casting.
\end{abstract}

Palavras-chave: alumina, freeze-casting, materiais porosos.

\begin{abstract}
Porous materials have attracted great attention as a new class of materials with a wide range of applications, including membranes for gas separation and desalination. It is well established that the properties of porous ceramic materials are related to the manufacturing process. Thus, the knowledge and control of the process parameters is a key issue for tailoring the properties of these materials. In this work alumina-based supports were prepared using tert-butyl alcohol as the solvent and citric acid as the dispersant. When the solid loading was increased from 15 to 30 vol\%, the total porosity of the alumina ceramic decreased linearly from 73 to $48 \%$. The samples showed a closed porosity below 2\%. The flexural strength of these materials ranged from $3.6 \pm 0.7$ to $21.9 \pm 0.6 \mathrm{MPa}$. The freeze-cast porous alumina was examined by scanning electron microscopy, and X-ray microtomography. The obtained samples exhibited a well-connected and ordered pore network. The results obtained in this work confirm the versatility of freeze-casting process for preparing porous ceramic materials.
\end{abstract}

Keywords: alumina, freeze-casting, porous materials.

\section{INTRODUÇÃO}

Materiais cerâmicos com porosidade controlada apresentam uma vasta gama de aplicações, valendo destacar filtros para remoção de inclusões em metais líquidos [1], isolantes térmicos $[2,3]$, matrizes para fabricação de compósitos [4-6] e biomateriais [7-9]. Além disso, em razão da crescente preocupação com a preservação do meio ambiente o uso de cerâmicas porosas em processos de separação gasosa e tratamento de águas residuais também é recorrente [10]. Nessas situações o desempenho do material é função tanto de sua estrutura de poros, quanto das propriedades intrínsecas (e.g., massa molecular, calor específico) e da fração volumétrica de sólidos. De fato, para que as propriedades de estruturas porosas sejam adequadas para determinada aplicação é necessário o rígido controle da morfologia, fração volumétrica, distribuição e tamanho de poros. Processos tradicionais de fabricação de cerâmicas porosas apresentam limitada capacidade em oferecer todos esses controles. Diante disso, um processo que tem despertado interesse nos últimos anos, devido à sua simplicidade, flexibilidade e utilização de matérias-primas ambientalmente amigáveis é o freeze-casting, ou moldagem por congelamento. Trata-se de uma técnica versátil que permite a obtenção de materiais com estrutura de poros controlada e propriedades anisotrópicas [11-15].

Tipicamente, suspensões utilizadas na conformação de materiais via freeze-casting apresentam concentração 
de sólidos variando entre 10 e $40 \%$ em volume (\%vol) e partículas com diâmetro médio entre $60 \mathrm{~nm}$ e $6,5 \mu \mathrm{m}$ [1316]. Está bem estabelecido [17] que o aumento da fração volumétrica de sólidos e a redução do tamanho médio das partículas dificultam a dispersão e a estabilização das suspensões cerâmicas, o que pode resultar em materiais com limitada orientação dos poros. Aspectos relativos às propriedades físico-químicas do solvente (e.g., viscosidade, constante dielétrica), partícula (e.g., densidade) e dispersante (e.g., polaridade, massa molecular) também devem ser considerados quando se deseja obter suspensões adequadas ao processo freeze-casting. Neste processo a suspensão cerâmica é vertida em um molde posicionado sobre uma superfície metálica cuja temperatura pode ser reduzida de maneira controlada. Durante a solidificação da suspensão ocorrem a segregação e a redistribuição das partículas devido ao avanço da frente de resfriamento, o que resulta na formação de duas regiões distintas. A primeira, rica em cristais de solvente, atua como template para a segunda região que é formada pelas partículas cerâmicas distribuídas entre esses cristais. Deville et al. [17, 18] afirmam que há duas condições básicas que devem ser atendidas para que haja formação de poros pelo processo freeze-casting. A primeira delas sugere que a energia livre interfacial entre as partículas e a frente de solidificação $\left(\sigma_{\mathrm{sp}}\right)$ deve ser maior do que a soma da energia livre interfacial do sólido-líquido $\left(\sigma_{\mathrm{sl}}\right)$ e da energia livre interfacial partícula-líquido $\left(\sigma_{\mathrm{pl}}\right)$. A outra condição está associada ao fato que a frente de resfriamento não pode ser plana, pois neste caso as partículas se concentrariam em apenas um dos lados da amostra.

Poros com diferentes morfologias são obtidos dependendo das características da suspensão, das condições de resfriamento, e do tipo de solvente. Em geral, a utilização de solventes tais como água, canfeno ou terc-butanol resulta em materiais com estruturas porosas dos tipos lamelares [19], dendríticas [20, 21] ou prismáticas [22, 23], respectivamente. Além disso, aditivos como glicerol [24], polietilenoglicol (PEG) [25], e acetato de polivinila (PVA) $[26,27]$ vêm sendo utilizados a fim de alterar a morfologia dos poros, o que tem garantido a obtenção de materiais com propriedades diferenciadas. As amostras solidificadas são liofilizadas, o que dá origem a uma estrutura de poros que é uma réplica dos cristais gerados durante o resfriamento. Posteriormente, consolidação do corpo verde é feita mediante tratamento térmico. Neste trabalho foi avaliado como a estrutura de poros de materiais cerâmicos à base de alumina pode ser controlada por meio do processo freezecasting. Os efeitos do dispersante e da concentração de sólidos sobre a microestrutura e resistência mecânica são objeto de investigação por meio de microscopia eletrônica de varredura (MEV), microtomografia de raios X $(\mu-\mathrm{CT})$ e ensaios de resistência à flexão.

\section{MATERIAIS E MÉTODOS}

Foram preparadas suspensões a partir da dispersão de diferentes concentrações $(15,20,25$ e $30 \%$ vol $)$ de alumina

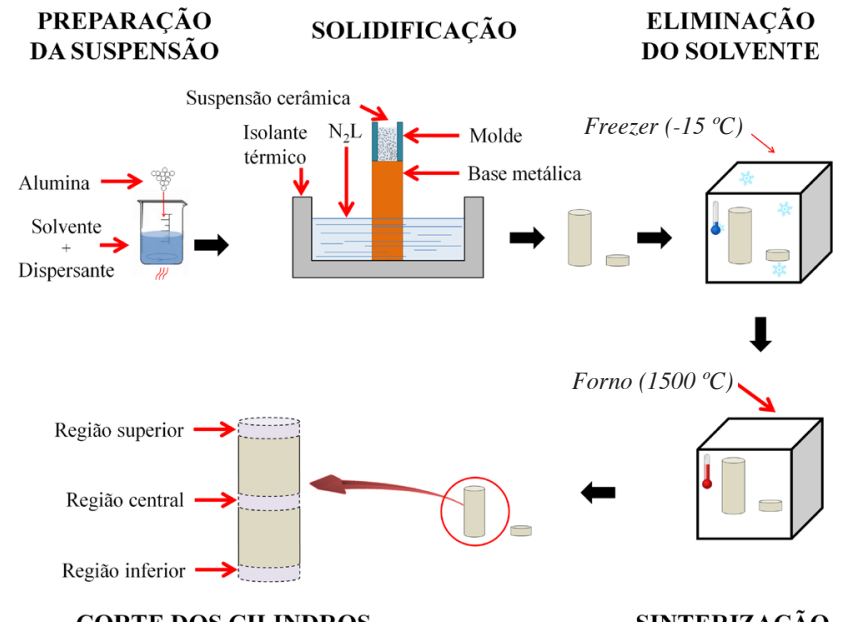

Figura 1: Representação esquemática das etapas adotadas na fabricação das amostras via freeze-casting.

[Figure 1: Schematic of the steps used in this work for preparing the freeze-cast samples.]

calcinada (CT3000SG, 99,8\%, Almatis Brasil) em solução de terc-butanol (TBA, $\geq 99,5 \%$, Aldrich) e ácido cítrico (AC, $\geq 99,5 \%$, Aldrich). A concentração de AC utilizada foi fixada em $0,5 \%$ em massa de alumina. Para verificar o efeito do dispersante sobre a microestrutura dos materiais, suspensões contendo $15 \% \mathrm{vol}$ de alumina foram preparadas sem a utilização de ácido cítrico. Depois de homogeneizadas, as suspensões foram ultrassonificadas por $5 \mathrm{~min}$ e, então, vertidas em moldes de acrílico posicionados sobre uma base metálica parcialmente imersa em nitrogênio líquido $\left(\mathrm{N}_{2} \mathrm{~L}\right)$. Após a completa solidificação, as amostras foram mantidas em um freezer doméstico a $15{ }^{\circ} \mathrm{C}$ por ao menos cinco dias, de modo a eliminar o solvente. Os corpos verdes foram tratados termicamente a $1500{ }^{\circ} \mathrm{C}$ entre 0 e $4 \mathrm{~h} \mathrm{em} \mathrm{um} \mathrm{forno}$ tipo mufla (Termolab). A taxa de aquecimento utilizada foi de $2{ }^{\circ} \mathrm{C} \cdot \mathrm{min}^{-1}$. A fim de verificar a evolução microestrutural ao logo do corpo de prova, as amostras cilíndricas $(12,5 \mathrm{~mm}$ x 25,0 $\mathrm{mm}$ ) preparadas foram seccionadas em três regiões (superior, central e inferior). Além disso, amostras na forma de pastilhas $(12,5 \mathrm{~mm} \times 2,0 \mathrm{~mm})$ também foram obtidas. A Fig. 1 exibe a representação esquemática do procedimento experimental adotado.

O efeito do dispersante foi verificado por meio de ensaios de distribuição de tamanho de partícula realizados em um granulômetro a laser (CILAS 1064). Ressalta-se que o TBA foi utilizado como solvente durante o ensaio. As amostras obtidas via freeze-casting foram examinadas por meio de microscopia eletrônica de varredura (MEV) e microtomografia de raios $\mathrm{X}(\mu-\mathrm{CT})$. Os ensaios de MEV foram realizados em equipamentos Aspex - PSEM Explorer e FEI QUANTA 200F. Antes desses ensaios, as amostras foram metalizadas com uma camada de ouro de aproximadamente $5 \mathrm{~nm}$. As análises de $\mu \mathrm{CT}$ foram realizadas em equipamento SkyScan 1172. As amostras investigadas foram rotacionadas em ângulos de até $180^{\circ}$, adotando-se passo de $0,30^{\circ}$. Mediante as projeções adquiridas e dos softwares NRecon e CTAn, obteve-se o índice de fragmentação definido por 
meio da Equação A [28]:

$$
\mathrm{IF}=\frac{\mathrm{S}_{1}-\mathrm{S}_{2}}{\mathrm{~V}_{1}-\mathrm{V}_{2}}
$$

onde, $\mathrm{S}$ e $\mathrm{V}$ são a superfície e o volume do sólido analisado, respectivamente, e 1 e 2 indicam o valor desses parâmetros antes e após a dilatação das imagens binarizadas, respectivamente.

Os valores médios foram acessados considerando-se um intervalo de confiança de $95 \%$. A resistência à flexão em três pontos das amostras obtidas neste trabalho foi obtida utilizando o acessório de ensaios mecânicos presente no equipamento de $\mu$-CT. Esses testes foram realizados conforme procedimento descrito na norma ASTM C167411. Amostras com dimensões de aproximadamente $5 \mathrm{~mm}$ x $5 \mathrm{~mm}$ x $15 \mathrm{~mm}$ foram utilizadas nesses ensaios. A resistência à flexão $(\sigma)$ dos materiais testados foi obtida por meio da Equação B:

$$
\sigma=\frac{3 \mathrm{Fl}}{2 \mathrm{bh}^{2}}
$$

onde, $F$ representa a carga crítica no momento da fratura, $l$ a distância entre os dois suportes do porta amostra, $b$ a espessura do material e $h$ a sua altura.

\section{RESULTADOS E DISCUSSÃO}

A Fig. 2 exibe a distribuição de tamanho de partículas para a alumina dispersa em terc-butanol. Quando ácido cítrico não foi utilizado, essa distribuição apresentou característica bimodal. Observam-se dois picos, um situado em $600 \mathrm{~nm}$ e outro em torno de $60 \mu \mathrm{m}$. Enquanto isso, a utilização de ácido cítrico reduziu de maneira significativa a presença de aglomerados; nota-se que a suspensão obtida com esse dispersante apresentou distribuição de tamanho de partículas monomodal centrado em torno de $1 \mu \mathrm{m}$. Por meio desse ensaio é possível afirmar que a utilização do dispersante contribuiu para a desaglomeração das partículas de alumina. Além disso, as micrografias apresentadas corroboram com os resultados obtidos a partir dos ensaios de granulometria a laser.

A Fig. 3 exibe micrografias de amostra preparada a partir de suspensão sem dispersante. Ressalta-se que a ausência de ácido cítrico resultou em suspensões com elevada viscosidade e, por isso, a concentração de alumina ficou limitada a $15 \%$ vol. Nota-se que a microestrutura obtida não é homogênea, sendo observada uma série de defeitos e aglomerados de partículas. Estes resultados sugerem que a presença dos aglomerados dificultou o crescimento dos cristais de terc-butanol durante a etapa de solidificação, prejudicando o controle da microestrutura do material preparado. Os resultados obtidos a partir dos ensaios de granulometria a laser e de MEV confirmam que a utilização de ácido cítrico é necessária, pois a ausência desse dispersante impede a produção de estruturas de poros ordenadas devido à presença dos aglomerados.
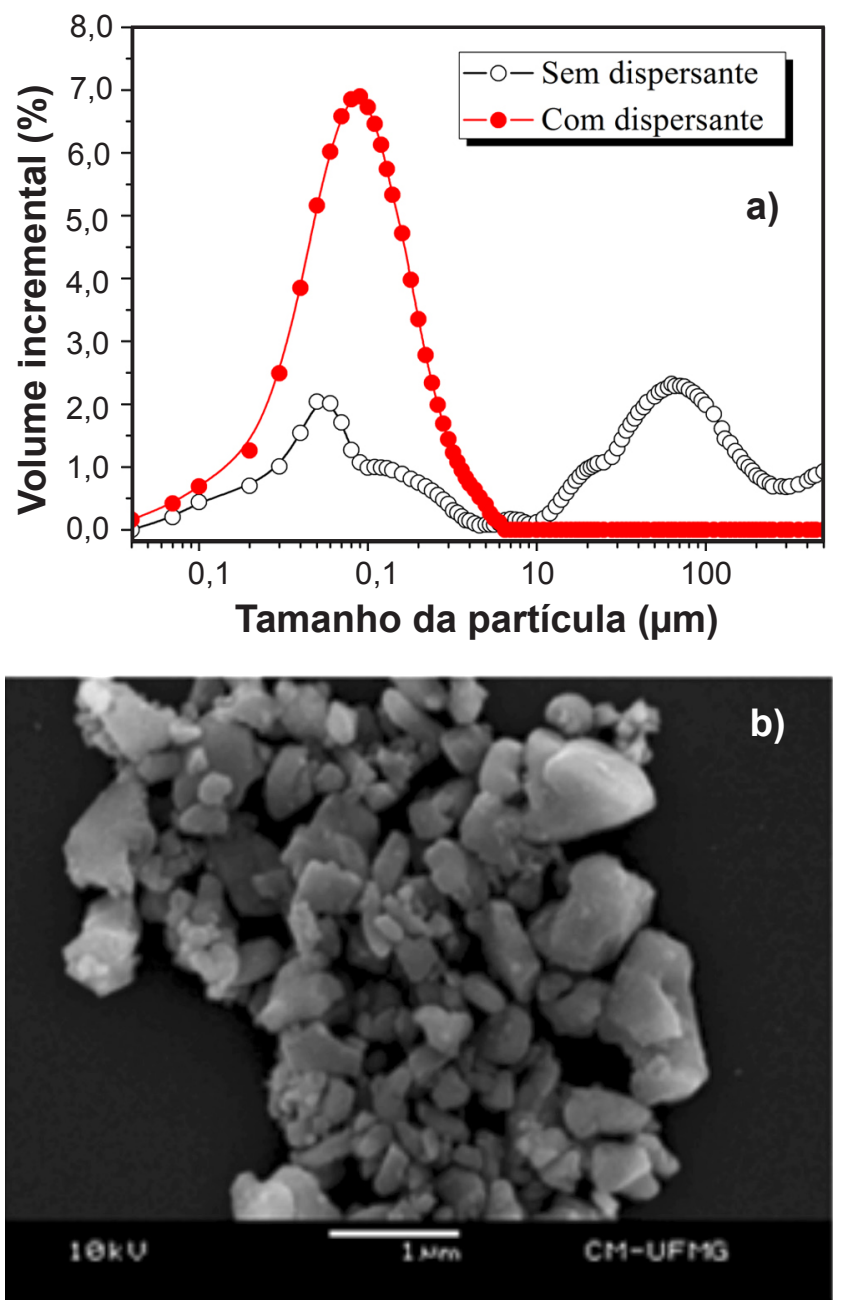

Figura 2: Distribuição de tamanho das partículas de alumina dispersas em TBA (a), e micrografia obtida por MEV (b).

[Figure 2: Particle size distribution of alumina powder dispersed in TBA (a), and SEM micrograph of alumina particles (b).]

As Figs. 4a a 4f exibem micrografias das superfícies paralelas à frente de solidificação de uma amostra cilíndrica fabricada a partir de suspensão contendo $30 \%$ vol de alumina (concentração máxima de alumina nas suspensões contendo ácido cítrico). Nota-se que, assim como nos trabalhos [29, 30], os materiais são constituídos principalmente por poros colunares com superfícies facetadas. Ressalta-se que na região inferior (Figs. 4e e 4f) uma camada aparentemente densa foi obtida. Uma análise mais detalhada revela que essa camada possui poros com pequenos diâmetros sem orientação definida. Por isso essa região foi classificada como isotrópica. De acordo com [13, 16], quando a velocidade da frente de solidificação supera a velocidade crítica, o rearranjo e a redistribuição das partículas são impedidos. Acredita-se que a elevada taxa de resfriamento próxima à base metálica tenha sido a responsável pela formação da camada isotrópica. Por outro lado, na medida em que a suspensão é solidificada a transferência de calor entre a base metálica e a suspensão decresce, o que resulta na redução da taxa de resfriamento da suspensão e, consequentemente, na diminuição da velocidade da frente de solidificação. Dessa 

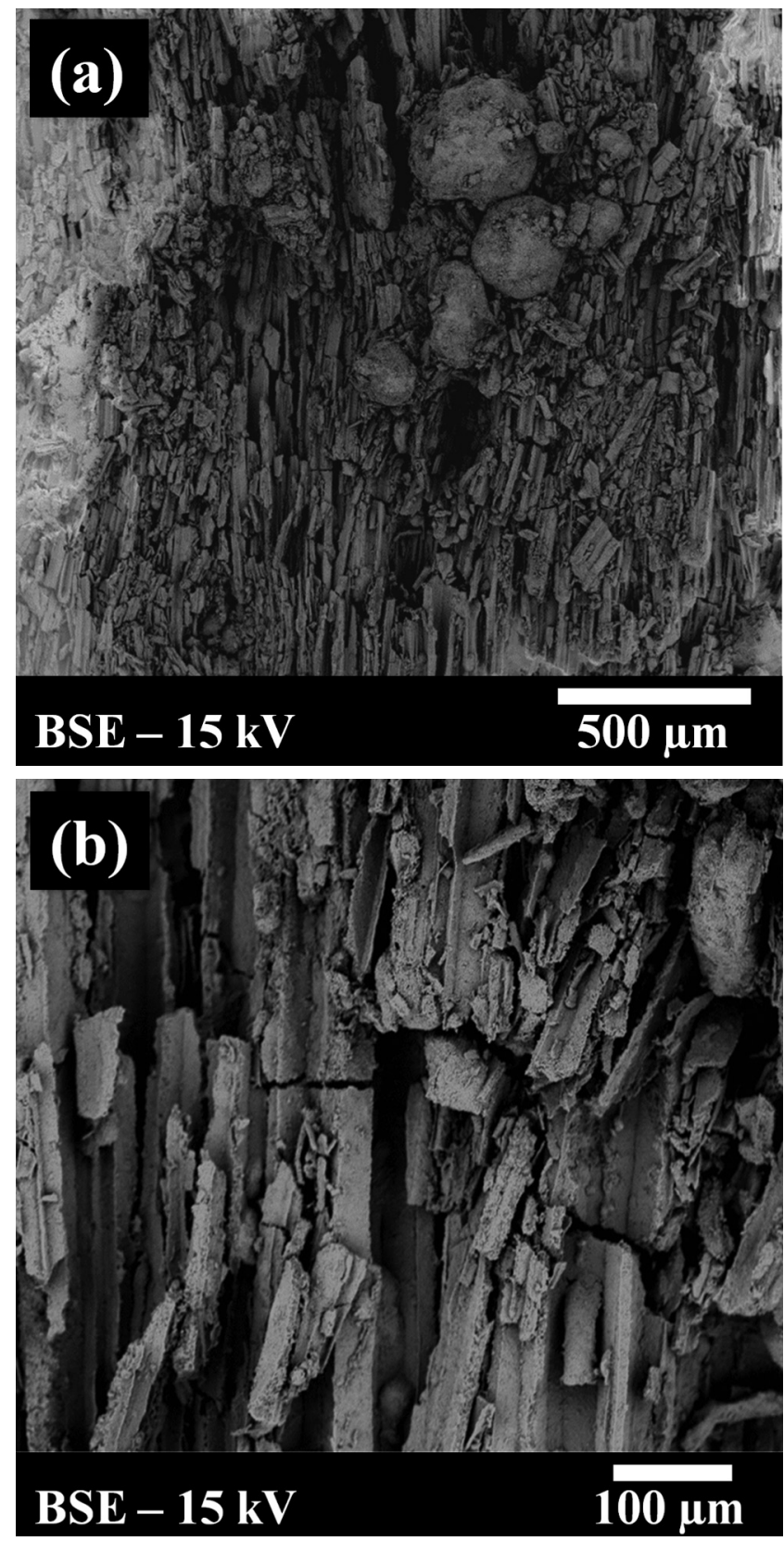

Figura 3: Micrografias obtidas por microscopia eletrônica de varredura de superfície paralela à frente de solidificação (amostra preparada sem dispersante).

[Figure 3: SEM micrographs of cross-sections parallel to the freezing direction (sample prepared without dispersant).]

forma, o crescimento orientado dos cristais de terc-butanol nas regiões superior (Figs. 4a e 4b) e central (Figs. 4c e 4d) é favorecido.

Devido à estrutura de poros observada na região inferior das amostras cilíndricas optou-se pela fabricação de amostras na forma de pastilhas $(12,5 \mathrm{~mm}$ x 2,0 mm). Acredita-se que materiais com esse tipo de estrutura possam atuar como suportes para membranas cerâmicas utilizadas em processos de separação gasosa, haja vista que nesse tipo de aplicação a presença de um gradiente de poros é necessária, a fim de facilitar tanto o transporte de fluidos quanto a deposição de camadas de separação [31, 32]. A Fig. 5 exibe micrografia da superfície paralela à frente de solidificação de uma pastilha obtida a partir de uma suspensão contendo $25 \%$ vol de alumina. Conforme esperado, é possível distinguir uma região isotrópica (indicada por setas) e uma região colunar. Os materiais obtidos neste estudo apresentaram gradiente de porosidade ao longo de sua espessura, o que poderia eliminar a necessidade de camadas intermediárias que geralmente são depositadas antes da camada de separação. Conforme será discutido mais adiante, materiais obtidos via freeze-casting apresentam elevada conectividade de poros. Assim, o transporte de massa na região colunar pode ser facilitado pela presença desses poros conectados.

A Tabela I apresenta o efeito do tempo de sinterização sobre o índice de fragmentação (IF) e a resistência à flexão dos materiais obtidos com diferentes tempos de sinterização. Os resultados referem-se às amostras obtidas a partir de suspensões contendo $25 \% \mathrm{vol}$ de alumina. O índice de fragmentação indica o grau de conectividade dos poros. Quanto menor o valor de IF, mais conectada é a estrutura de poros [33]. Em contrapartida, um alto valor de IF representa uma rede de poros desconexa. Nota-se que o aumento do tempo de sinterização resulta em amostras com maiores índices de fragmentação. Com isso, amostras sinterizadas por tempos mais longos exibem estruturas de poros mais desconexas, o que pode influenciar o transporte de fluidos através do material. Enquanto isso, o aumento do tempo de sinterização de zero, ou seja, sem patamar de sinterização, para $1 \mathrm{~h}$ resultou em amostras com maior resistência à flexão. Entretanto, um posterior aumento do tempo de sinterização parece não alterar de forma significativa a resistência à flexão das amostras.

Embora apresente uma conectividade de poros menor quando comparada à amostra sem patamar de sinterização, a amostra sinterizada durante $1 \mathrm{~h}$ possui resistência à flexão equivalente às amostras sinterizadas durante 2 ou $4 \mathrm{~h}$, indicando que maiores tempos de sinterização não seriam necessários. Com base nos resultados alcançados, acreditase que as amostras sinterizadas durante $1 \mathrm{~h}$ apresentam um melhor compromisso entre resistência mecânica e transporte de fluidos. Desta forma, determinou-se que as amostras obtidas a partir de suspensões com diferentes concentrações de alumina seriam sinterizadas durante $1 \mathrm{~h}$. A Fig. 6 apresenta os resultados obtidos para as porosidades total e fechada, determinadas conforme a norma ASTM B962-13, que é baseada no princípio de Archimedes. Notase que a porosidade total dos suportes diminui de maneira aproximadamente linear à medida que a concentração de sólidos na suspensão é aumentada. Enquanto isso, a porosidade fechada foi inferior a $2 \%$ para qualquer concentração de sólidos. É importante ressaltar que a baixa fração de poros fechados é um importante resultado, visto que os mesmos não contribuem para o transporte de fluidos.

A Tabela II exibe o efeito da concentração de sólidos sobre o índice de fragmentação e resistência à flexão dos materiais sinterizados durante $1 \mathrm{~h}$. Observa-se o aumento do IF quando a concentração de alumina em suspensão também 

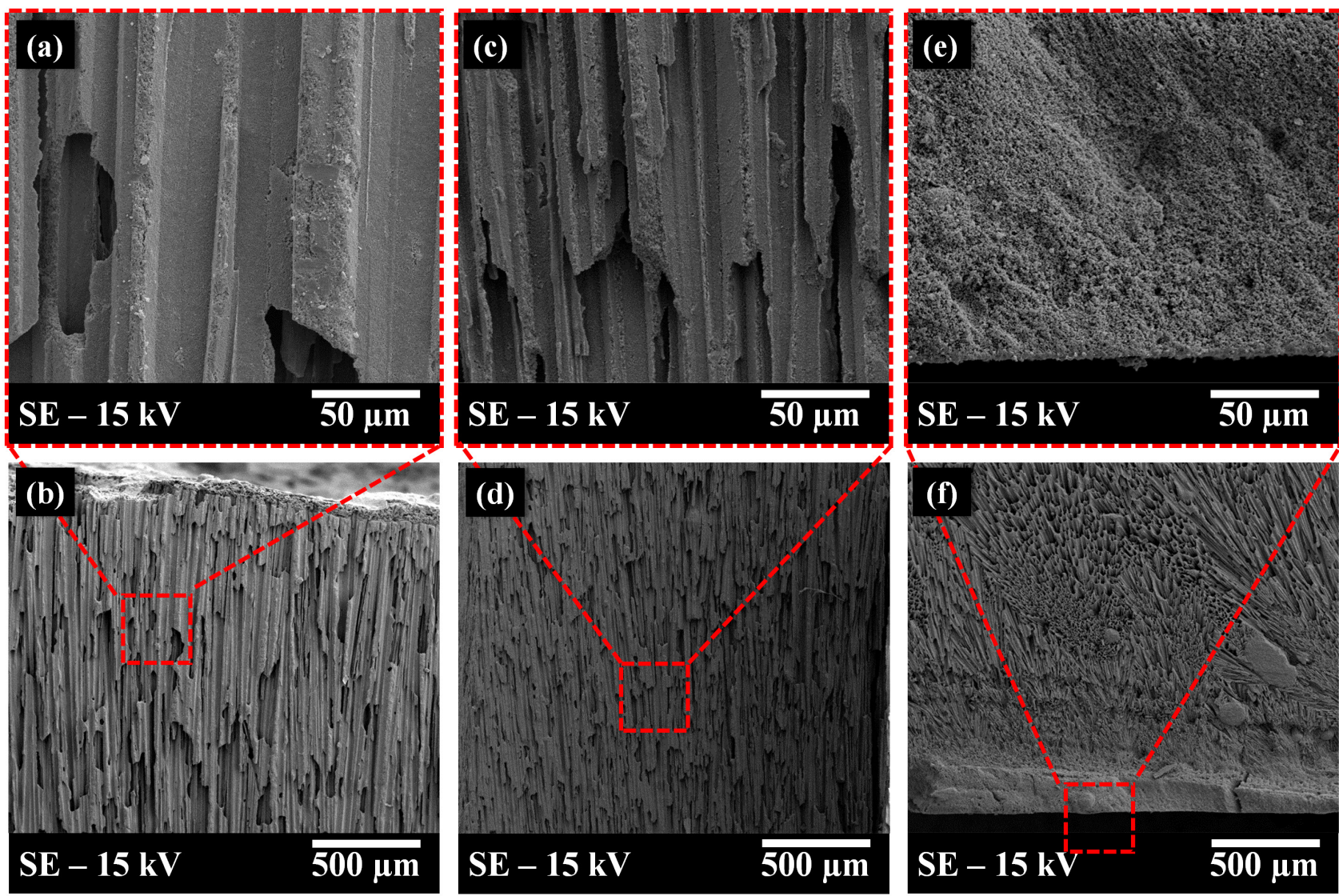

Figura 4: Micrografias obtidas por microscopia eletrônica de varredura da superfície paralela à frente de solidificação: (a, b) região superior, (c, d) região central, (e, f) região inferior. Amostra fabricada a partir de suspensão contendo 30 \%vol de alumina (com dispersante).

[Figure 4: SEM micrographs of cross-sections parallel to the freezing direction: $(a, b)$ top, $(c, d)$ middle, and (e, f) bottom regions. Sample prepared by keeping the alumina concentration constant at $30 \mathrm{vol} \%$ (with dispersant).]

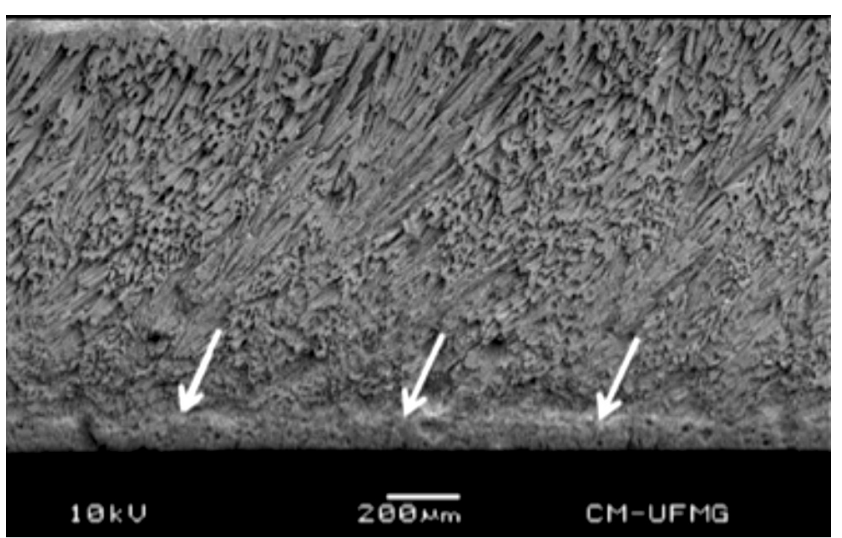

Figura 5: Micrografia obtida por microscopia eletrônica de varredura da superfície paralela à frente de resfriamento.

[Figure 5: SEM micrograph of cross-section parallel to the freezing direction.]

é aumentada. Sabe-se que após a sublimação dos cristais de solvente a porosidade das amostras preparadas é uma réplica direta da estrutura desses cristais. Portanto, quanto maior a concentração de solvente na suspensão cerâmica, maior é a porosidade do material obtido e também a conectividade dos poros. Esse comportamento está em acordo com os resultados descritos neste trabalho. Além disso, foi
Tabela I - Efeito do tempo de sinterização sobre o índice de fragmentação (dados obtidos por meio de $\mu$-CT) e resistência à flexão das amostras.

[Table I - Effect of the sintering time on fragmentation index (results obtained by $\mu-C T$ ) and the flexural strength of samples.]

\begin{tabular}{ccc}
\hline $\begin{array}{c}\text { Tempo de } \\
\text { sinterização }(\mathrm{h})\end{array}$ & $\mathrm{IF}\left(\mu \mathrm{m}^{-1}\right)$ & $\begin{array}{c}\text { Resistência à flexão } \\
(\mathrm{MPa})\end{array}$ \\
\hline 0 & $-0,101 \pm 0,001$ & $9,4 \pm 1,1$ \\
1 & $-0,077 \pm 0,001$ & $16,4 \pm 1,2$ \\
2 & $-0,066 \pm 0,001$ & $14,9 \pm 1,2$ \\
4 & $-0,033 \pm 0,001$ & $14,7 \pm 1,4$ \\
\hline
\end{tabular}

observado que quanto maior é a concentração de sólidos na suspensão, maior é a resistência à flexão do material obtido. Este comportamento está associado à diminuição da porosidade das amostras à medida que a concentração de alumina é aumentada. Embora apresentem maior resistência à flexão, as amostras obtidas a partir de suspensão contendo 30 \%vol de sólidos possuem viscosidade elevada, o que dificulta sua utilização. Suspensões contendo $25 \%$ vol de alumina apresentam melhor trabalhabilidade e, além disso, dão origem a materiais com microestrutura e resistência à 
Tabela II - Efeito da concentração de alumina sobre o índice de fragmentação e resistência à flexão de amostras.

[Table II - Effect of the alumina concentration on fragmentation index and the flexural strength of samples.]

\begin{tabular}{ccc}
\hline $\begin{array}{c}\text { Concentração de } \\
\text { alumina }(\% \text { vol })\end{array}$ & IF $\left(\mu \mathrm{m}^{-1}\right)$ & $\begin{array}{c}\text { Resistência à } \\
\text { flexão }(\mathrm{MPa})\end{array}$ \\
\hline 15 & $-0,131 \pm 0,002$ & $3,6 \pm 0,7$ \\
20 & $-0,132 \pm 0,002$ & $5,6 \pm 0,5$ \\
25 & $-0,077 \pm 0,001$ & $16,4 \pm 1,2$ \\
30 & $-0,053 \pm 0,001$ & $21,9 \pm 0,6$ \\
\hline
\end{tabular}

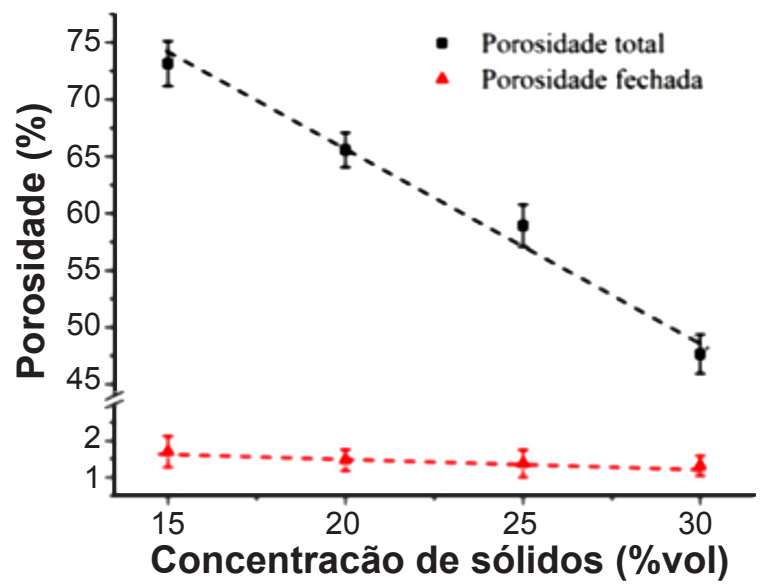

Figura 6: Efeito da concentração de alumina sobre a porosidade das amostras. As linhas são apenas guias para os olhos.

[Figure 6: Effect of the alumina concentration on the samples porosity. The lines are used only as a guide to the eyes.]

flexão adequadas. Vale mencionar que ensaios de permeação realizados nas amostras obtidas neste estudo apontaram que materiais processados via processo freeze-casting podem ser utilizados como suportes para fabricação de membranas cerâmicas empregadas em processos de separação gasosa. Esses testes estão apresentados no trabalho [23].

\section{CONCLUSÕES}

Neste estudo as amostras de alumina preparadas via processo freeze-casting apresentaram duas regiões distintas; a primeira próxima à superfície de resfriamento foi denominada isotrópica, e é formada por poros sem orientação aparente. A segunda região é formada por poros colunares preferencialmente orientados em direção paralela ao gradiente térmico gerado durante a etapa de solidificação. $\mathrm{O}$ controle adequado das condições de processamento permitiu a obtenção de materiais livres de defeitos (aglomerados, trincas), e com elevada porosidade aberta (> 45\%). Cálculos baseados nos ensaios de $\mu$-CT apontam que as amostras exibem estruturas de poros bem conectadas o que a princípio pode facilitar o transporte de fluidos através desses materiais. Os resultados alcançados apontam que o processo freeze-casting é bastante promissor para a fabricação de materiais com estruturas de poros bem definidas com elevada conectividade de poros.

\section{AGRADECIMENTOS}

Os autores agradecem ao $\mathrm{CNPq}$, Petrobras, Statoil Brasil Óleo e Gás e ANP (Agência Nacional do Petróleo, Gás Natural e Biocombustíveis) pelo apoio financeiro a essa pesquisa. Também a Almatis do Brasil pelo fornecimento das partículas de alumina utilizadas nesse estudo.

\section{REFERÊNCIAS}

[1] E. Storti, M. Emmel, S. Dudczig, P. Colombo, C.G. Aneziris, J. Eur. Ceram. Soc. 35 (2015) 1569.

[2] E. Bartonickova, P. Ptacek, T. Opravil, F. Soukal, J. Masilko, R. Novotny, J. Svec, J. Havlica, Ceram. Int. 41 (2015) 14116.

[3] T. Shimizu, K. Matsuura, H. Furue, K. Matsuzak, J. Eur. Ceram. Soc. 33 (2013) 3429.

[4] M.E. Launey, E. Munch, D.H. Alsem, E. Saiz, A.P. Tomsia, R.O. Ritchie, J. R. Soc. Interface 7 (2010) 741.

[5] J. Wang, J. Qiao, J. Wang, Y. Zhu, L. Jiang, ACS Appl. Mater. Interfaces 7 (2015) 9281.

[6] S. Roya, J. Gibmeier, V. Kostov, K.A. Weidenmann, A. Nagelb, A. Wanner, Acta Mater. 59 (2011) 1424.

[7] S. Deville, E. Saiz, A.P. Tomsia, Biomater. 27 (2006) 5480 .

[8] Q. Fu, E. Saiz, M.N. Rahaman, A.P. Tomsia, Adv. Funct. Mater. 23 (2013) 5461.

[9] X. Liu, M.N. Rahaman, Q. Fu, A.P. Tomsia, Acta Biomater. 8 (2012) 415.

[10] E.C. Hammel, O.L.-R. Ighodaro, O.I. Okoli, Ceram. Int. 40 (2014) 15351.

[11] S. Deville, Mater. 3 (2010) 1913.

[12] T. Fukasawa, M. Ando, T. Ohj, S. Kanzaki, J. Am. Ceram. Soc. 1 (2001) 230.

[13] W.L. Li, K. Lu, J.Y. Walz, Int. Mater. Rev. 57 (2012) 37.

[14] Z. Hou, F. Yeb, L. Liu, J. Eur. Ceram. Soc. 35 (2015) 4115.

[15] J. Verma, M. Vijayakumar, R. Mitra, Mater. Lett. 153 (2015) 168.

[16] S. Deville, Adv. Eng. Mater. 10 (2008) 155.

[17] S. Deville, E. Maire, G. Bernard-Granger, A. Lasalle, A. Bogner, C. Gauthier, J. Leloup, C. Guizard, Nat. Mater. 8 (2009) 966.

[18] S. Deville, E. Saiz, A.P. Tomsia, Acta Mater. 55 (2007) 1965.

[19] Y. Tang, Q. Miao, S. Qiu, K. Zhao, L. Hu, J. Eur. Ceram. Soc. 34 (2014) 4077.

[20] A.M.A. Silva, E.H.M. Nunes, D.F. Souza, D.L. Martens, J.C. Diniz da Costa, M. Houmard, W.L. Vasconcelos, Ceram. Int. 41 (2015) 10467.

[21] R. Sepúlveda, A.A. Plunk, D.C. Dunand, Mater. Lett. 142 (2015) 56.

[22] M. Naviroj, S.M. Miller, P. Colombo, K.T. Faber, J. 
Eur. Ceram. Soc. 35 (2015) 2225.

[23] D.F. Souza, E.H.M. Nunes, D.S. Pimenta, D.C.L. Vasconcelos, J.F. Nascimento, W. Grava, M. Houmard, W.L. Vasconcelos, Mater. Charact. 96 (2014) 183.

[24] Y. Zhang, Ceram. Int. 36 (2010) 617.

[25] C. Pekor, I. Nettleship, Ceram. Int. 40 (2014) 171.

[26] K. Lebreton, J.M. Rodríguez-Parra, R. Moreno, M.I. Nieto, Adv. Appl. Ceram. 114 (2015) 296.

[27] C. Pekor, B. Groth, I. Nettleship, J. Am. Ceram. Soc. 93 (2010) 115.

[28] M. Hahn, M. Vogel, M. Pompesius-Kempa, G. Delling, Bone 13 (1992) 327.

[29] H.J. Choi, T.Y. Yang, S.Y. Yoon, B.K. Kim, H.C. Parka, Mater. Chem. Phys. 133 (2012) 16.
[30] R. Chen, C.-A. Wang, Y. Huang, L. Ma, W. Lin, J. Am. Ceram. Soc. 90 (2007) 3478.

[31] H.J.M. Bouwmeester, A.J. Burggraaf, Fundamentals of Inorganic Membrane Science and Technology, Elsevier, Amsterdam (1996) 435.

[32] A.J. Burggraaf, K. Keizer, Synthesis of inorganic membranes, In: R. R. Bhave, Inorganic Membranes: synthesis, characteristics and applications, Van Nostrand Reinhold, New York (1991) 10.

[33] L.L.O. Silva, D.C.L. Vasconcelos, E.H.M. Nunes, L. Caldeira, V.C. Costa, A.P. Musse, S.A. Hatimondi, J.F. Nascimento, W. Grava, W.L. Vasconcelos, Ceram. Int. 38 (2012) 1943.

(Rec. 17/02/2016, Rev. 03/03/2016, Ac. 04/05/2016) 